\title{
sciendo
}

\author{
BULGARIAN ACADEMY OF SCIENCES
}

CYBERNETICS AND INFORMATION TECHNOLOGIES • Volume 19, No 1

Sofia $2019 \quad$ Print ISSN: 1311-9702; Online ISSN: 1314-4081

DOI: $10.2478 /$ cait-2019-0011

\section{Application of Information Technologies and Algorithms in Ship Passage Planning}

\section{Blagovest Belev, Dilyan Dimitranov, Alexandar Spasov, Aleksandar Ivanov}

Nikola Vaptsarov Naval Academy, Faculty of Navigation, 73 Vasil Drumev Str, Varna, Bulgaria E-mails:b.belev@nvna.eu dimitranov@gmail.com alexandyr.spasov@gmail.com

\begin{abstract}
With the continuous increase of international oil prices, more and more shipping companies look for new solutions to the ever present question: How to reduce operational fuel consumption and decrease air pollution. Ship route planning is an indispensable part of the ship navigation process. In the modern world, the passage planning aspect of navigation is shifting. No longer do we see mariners drawing course lines on a paper chart. No longer do they calculate distances with compasses. Elaborate algorithms on various digital devices perform all these tasks. Algorithms plot the optimum tracks on digital charts and algorithms can decide how to avoid collision situations. Nowadays charter companies do not rely solely on the experienced navigators on board their vessels to decide the best route. Instead, this task is outsourced ashore to routing and weather-routing enterprises. The algorithms used by those enterprises are continuously evolving and getting better and better. They are coming popular because of another reason - more and more the shipping society support the newly idea for using crewless ships. However, are they up to the task to eliminate the human element in passage planning? In this article, we are going to review some of the weak points of the algorithms in use.
\end{abstract}

Keywords: Information technologies in shipping, cybernetic decisions, evolutionary algorithm, safety of navigation.

\section{Introduction}

Safety in shipping is a priority task regardless of the time and technology development. Many researchers are attempting to use different mathematical algorithms that adapt to shipping conditions. Modern information and computing technologies make it possible to implement any scientific idea. A. Kumar, D. Kumar, and S. Jarial [1] have analysed a number of algorithms and the possibilities for their application in solving data clustering problems. Their study presents an in-depth study of swarm intelligence techniques and is an example of a research approach to the algorithms used in other scientific fields. 
Nowadays in the shipping a new breed of algorithms is emerging - the Evolutionary Algorithms (EAs). As B a l a b a n o v, Zankinski and B arova [2] have noted EAs are applied successfully in finding acceptable solutions to problems in business, engineering, and science.

The common underlying idea behind the evolutionary algorithms is the same as in the evolutionary processes in nature: Given a population of individuals, the environmental pressure causes natural selection (survival of the fittest) and this causes a rise in the fitness of the population [3].

The idea of applying the principles of Neo-Darwinism for automatic problem solving emerged in the $30 \mathrm{~s}$ of the 20th century [4]. In the $60 \mathrm{~s}$ and $70 \mathrm{~s}$, several scientists developed different applications of the main ideas at the same time. In the United States, Vogel, Owens and Walsh present evolutionary programming [5], while Holland calls its method a genetic algorithm [6]. At the same time, in Germany, Rahlenberg and Schweifel create theirs evolutionary strategies [7]. In the next two decades, these areas have evolved separately, but since the $90 \mathrm{~s}$, they have begun to perceive themselves as different representatives of a technology called evolutionary computing.

Given a quality function to be maximized, a set of candidate solutions can be randomly created and the quality function as an abstract fitness measure can be applied. Based on this fitness, some of the better candidates are chosen to seed the next generation by applying recombination and/or mutation to them [9].

Evolutionary Algorithms (from here on referenced as EAs) are algorithms that can perform optimization tasks and have the inherent ability to evolve. In general, they have three main characteristics:

- Population-based. EAs support a selection of solutions, called a population, to optimize the problem. The population is a basic principle of the evolutionary process.

- Fitness-oriented. Every solution in a population is called an individual. Every individual has its gene representation, called its code, and performance evaluation, called its fitness value. EAs choose fitter individuals, and that is the foundation of the optimization and convergence of the algorithms.

- Variation-driven. Individuals will sustain a number of variations to imitate genetic changes, which is the basis for searching the solution space.

EAs, applied for ship passage planning, can be divided into three types:

- Genetic,

- Ant Colony Algorithms,

- Particle Swarm Algorithms.

In this article, we will look more closely into the first type of algorithms - the genetic evolutionary algorithms.

\section{Genetic evolutionary algorithms - brief overview}

Evolution via natural selection of a randomly chosen population of individuals can be thought of as a search through the space of possible chromosome values. In that 
sense, a Genetic Evolutionary Algorithm (Genetic EAs) is a stochastic search for an optimal solution to a given problem.

The evolutionary search process is influenced by the following main components of and EA:

- An encoding of solutions to the problem as a chromosome;

- A function to evaluate the fitness of the individuals;

- Setup of the initial population;

- Selection operators;

- Reproduction operators.

Evolutionary algorithms can be used for both weather routing and collision avoidance. However, Genetic EAs can be particularly effective in collision avoidance situations and in plotting the safe passage through coastal waters. An example of that are the works of Szlapczynski [6], Szlapczynski and Szlapczynska [7], and Ge org i e va [8]. Avoiding collision and choosing the shortest and safest path are two steps towards achieving higher energy efficiency. Moreover, the obvious question is: Are Genetic EAs ready to be implemented on board vessels?

In the following section, some limitations and constraints to their immediate use for passage planning and collision avoidance will be reviewed.

\subsection{Limitations to Genetic EAs with regard to on-board use}

\subsubsection{Issues with Automatic Identification System (AIS)}

When collecting input data, most EAs process the information from the AIS or the Automatic Radar Plotting Aid (ARPA). This is a good approach as ship motion parameters can be obtained directly from either system with a varying degree of accuracy. However, some algorithms may have the option to read out the type of ship from the AIS. Others may have the function to acquire the length of other vessels from the AIS. These functions are present so that an EA can determine the opponent ship's domain in order to provide a safe passing. This approach, as convenient as it is, may lead either to an error in the solution provided by the algorithm or to its complete collapse. The reason for this is two-fold. Firstly when AIS targets are obtained by the AIS, at the beginning only the most relevant data is obtained, and that is the dynamic data: Target's position, course and speed, which is transmitted every $2-10 \mathrm{~s}$ (depending on ship's speed and rate of turn). Static data is transmitted every 6 minutes. Because of the time gap, an algorithm may provide an error in its solution if the target has just appeared. A good way to eliminate this problem would be for the algorithm to assume a default length of the vessel, until the target's attributes can be properly acquired. This proposal would also benefit from those vessels, which have not inputted any ship specific data in the AIS, such as small fishing boats, or vessels equipped with AIS type B.

Another point for consideration, where algorithms provide the functionality of collision avoidance, should be made when the status of opponent vessels is different from "Under way using engine". If a read-out of the AIS static data is introduced in the algorithm, it should be able to differentiate between the various navigational statuses and adjust the fitness function accordingly. In this way it will be benefiting individuals which violate the COLlision REGulations (COLREG) the least and 
penalizing those which generate a solution, where own ship does not behave as a give-way vessel where it should.

\subsubsection{Issues with Electronic Chart Display and Information System (ECDIS)}

For an algorithm to work as intended, to avoid other ships and all navigational dangers, it must be able to acquire a proper interpretation of the area around the vessel effectively. In his research for the evolutionary computing and multi-trajectory, planning R. S z la p c z y n ski [6] uses bitmaps, generated off vector maps in order to limit the time needed to process the vector maps as they consume too much time for data read-out. Such bitmaps are generated offline and stored on board, so they can be easily accessed when the algorithm is running in real time.

This design may be considered ineffective because ECDIS worldwide are updated on a weekly basis. If a vessel uses bitmaps for the calculation purposes then either it must have the conversion software on board or it must have a very good internet connection in order to receive the bitmaps from a shore company. In either case, this is not an ideal solution because:

- Having the software on board means that a good amount of time will be consumed for the new vector charts to be converted to bitmaps, thus newly updated cells cannot be used immediately. On top of that, distributing licensed software to all vessels in a company may turn out to be rather expensive.

- Internet speed on board most trade vessels is still relatively slow. Thus dispatching big files to the vessel may turn out to be quite cumbersome.

A more elegant solution would be to use an object's attributes from the ECDIS. A new attribute can be included for each ECDIS object, which can define a value for a safe passing distance, depending on the danger that attribute represents. Values can range from 0 up to 10 . Because vessels have varying design, the safe passing distance can be a function of the vessel's Advance, with the value of the ECDIS object as a coefficient in a formula.

For preliminary calculations, the following formulas can be used to calculate the Advance of a given ship. First, a steady tactical diameter (in ship lengths) has to be calculated as

(1) $\begin{aligned} \frac{\mathrm{STD}}{L} & =4.19-203 \frac{C_{B}}{\delta_{R}}+47.4 \frac{\mathrm{Trim}}{L}-\frac{13.0 B}{L}+\frac{194}{\delta_{R}}-35.8 \frac{\mathrm{Sp} \times \mathrm{Ch}}{L \times T}(\mathrm{ST}-1)+ \\ & +3.82 \frac{\mathrm{Sp} \times \mathrm{Ch}}{L \times T}(\mathrm{ST}-2)+7.79 \frac{A_{B}}{L \times T}+0.7\left(\frac{T}{T_{L}}-1\right)\left(\frac{\delta_{R}}{\left|\delta_{R}\right|}\right)(\mathrm{ST}-1),\end{aligned}$ where:

STD - Steady Turning Diameter, m;

$C_{B} \quad$ - Block Coefficient;

$\delta_{R} \quad$ - Rudder angle, degrees (positive to starboard);

Trim - static Trim, m;

$L \quad$ - Length of the vessel, measured between perpendiculars, m;

B - moulded Breadth, m;

Sp $\quad-$ Span of rudder, $\mathrm{m}$;

$\mathrm{Ch} \quad$ - mean Chord of rudder, $\mathrm{m}$;

$T \quad$ - design draft at full load, $m$;

ST - Stern Type (1 - Closed, 2 - Open $)$; 
$T_{L} \quad-$ draft, at which Turning circle is estimated, m;

$A_{B} \quad$ - submerged Bow profile Area, $\mathrm{m}^{2}$;

$$
\begin{gathered}
\frac{\mathrm{TD}}{L}=0.910 \frac{\mathrm{STD}}{L}+0.424 \frac{\mathrm{Vs}}{\sqrt{L}}+0.675, \\
\frac{\mathrm{Ad}}{L}=0.519 \frac{\mathrm{TD}}{L}+1.33
\end{gathered}
$$

where

$\mathrm{TD}=$ Tactical Diameter, $\mathrm{m}$;

$\mathrm{Vs}=$ Vessel speed, in knots,

$\mathrm{Ad}=$ Advance, in $\mathrm{m}$ [3].

The formula for safe distance around the vessel can be as follows:

$$
\mathrm{SD}=\frac{\mathrm{Vs} \times \mathrm{Ad}^{2} \times \sqrt{\mathrm{Av}}}{300},
$$

where

$\mathrm{SD}=$ Safe Distance, in $\mathrm{nm}$,

Vs = Vessel speed, knots;

Ad = Advance, $\mathrm{nm}$;

$\mathrm{Av}=\mathrm{ENC}$ Attribute safety value.

For a given ship with the following characteristics:

Length between perpendiculars, $L$,

$349.8 \mathrm{~m}$,

Breadth molded, $B$,

$58.3 \mathrm{~m}$,

Draft in full load, $T$,

$19.4 \mathrm{~m}$,

Block coefficient,

0.875 ,

Displacement, metric tons,

355,600 ,

Speed, corresponding to $85 \%$ of engine output, Vs, 15 knots,

Movable Rudder Area, AR,

Rudder Chord, Ch,

$164.8 \mathrm{~m}^{2}$,

$10.8 \mathrm{~m}$,

Rudder Span, Sp,

$15.2 \mathrm{~m}$,

Rudder deflection rate, $\mathrm{r} \delta$,

Submerged Area of Bow profile, AB,

Stern Type (1 - closed, 2 - open), ST,

Number or propellers,

$0.04 \mathrm{rad} / \mathrm{s}$,

$8 \mathrm{~m}^{2}$,

1 ,

1.

Assuming rudder angle at $35^{\circ}$ and no trim, the steady turning diameter in ship lengths will be

$$
\frac{\mathrm{STD}}{L}=2.408 \text {. }
$$

And the tactical diameter and the advance (in ship lengths) will be

$$
\frac{\mathrm{TD}}{L}=3.206, \frac{\mathrm{Ad}}{L}=2.994 \text {, and } \mathrm{Ad}=0.565 \mathrm{~nm} .
$$

Depths are already implemented as objects in an ENC and can be directly used by the algorithm. This solution could eliminate the above mentioned disadvantages using bitmaps. However at this moment, there is not a solution that can be deemed perfect since the direct read-out of the attributes may consume more time for calculations. Yet with the advancement in technology this particular disadvantage can be considerably mitigated. 
Table 1. Table 1 and graph on Fig. 1 represent how the safety distance changes with the change of the vessel's speed and with the increasing of the ENC safety value, in $\mathrm{m}$

\begin{tabular}{|c|c|c|c|c|c|c|c|c|c|c|}
\hline \multirow{2}{*}{$\begin{array}{c}\text { Vessel } \\
\text { speed, knots }\end{array}$} & \multicolumn{10}{|c|}{ Distans change, $\mathrm{m}$} \\
\cline { 2 - 12 } & 1 & 2 & 3 & 4 & 5 & 6 & 7 & 8 & 9 & 10 \\
\hline 2 & 13 & 19 & 23 & 27 & 30 & 32 & 35 & 37 & 40 & 42 \\
\hline 4 & 53 & 76 & 93 & 107 & 120 & 131 & 141 & 151 & 160 & 169 \\
\hline 6 & 121 & 171 & 210 & 242 & 271 & 297 & 321 & 343 & 364 & 383 \\
\hline 8 & 217 & 307 & 376 & 435 & 486 & 532 & 575 & 615 & 652 & 687 \\
\hline 10 & 342 & 484 & 593 & 684 & 765 & 838 & 906 & 968 & 1027 & 1082 \\
\hline 12 & 497 & 703 & 860 & 994 & 1111 & 1217 & 1314 & 1405 & 1490 & 1571 \\
\hline 14 & 682 & 964 & 1181 & 1363 & 1524 & 1669 & 1803 & 1928 & 2045 & 2155 \\
\hline 16 & 897 & 1269 & 1554 & 1794 & 2006 & 2198 & 2374 & 2538 & 2692 & 2837 \\
\hline 18 & 1144 & 1618 & 1982 & 2289 & 2559 & 2803 & 3028 & 3237 & 3433 & 3619 \\
\hline 20 & 1424 & 2014 & 2466 & 2848 & 3184 & 3488 & 3767 & 4027 & 4272 & 4503 \\
\hline 22 & 1736 & 2455 & 3007 & 3472 & 3882 & 4253 & 4593 & 4911 & 5208 & 5490 \\
\hline 24 & 2082 & 2944 & 3606 & 4164 & 4655 & 5100 & 5508 & 5889 & 6246 & 6584 \\
\hline
\end{tabular}

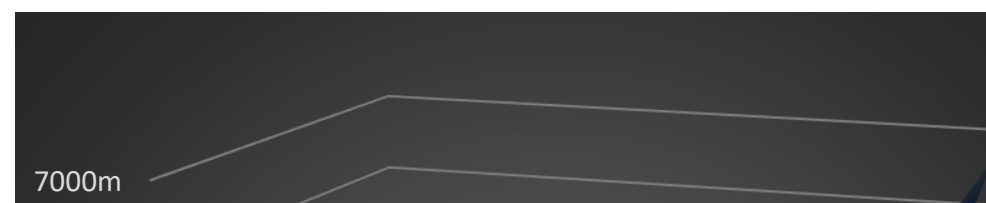

$7000 m$

$6000 \mathrm{~m}$

$5000 \mathrm{~m}$

$4000 m$

$3000 \mathrm{~m}$

$2000 \mathrm{~m}$

$1000 \mathrm{~m}$

Om

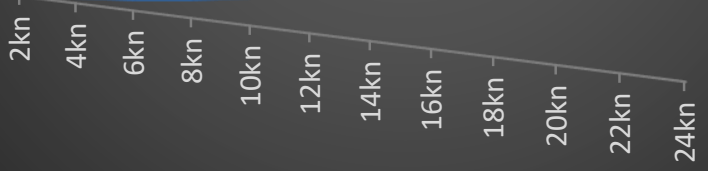

- 0m-1000m $\quad 1000 m-2000 m \square 2000 m-3000 m=3000 m-4000 m$

$4000 \mathrm{~m}-5000 \mathrm{~m}=5000 \mathrm{~m}-6000 \mathrm{~m}-6000 \mathrm{~m}-7000 \mathrm{~m}$

Fig.1. Representation of how Safety distance changes comparatively to ship's speed and ENC Safety value (in $\mathrm{m}$ ) 


\subsubsection{The problem with ship domains}

Nowadays the problem with the development of ship domains is that they are single purposed. They only represent the safe distance around the vessel for passing a singular type of obstacle. Most commonly this obstacle is another ship, thus most ship domains are designed for safe passing with other vessels. There has been a lot of research on the subject of ship domains, but almost none have examined the idea of multiple ship domains overlaying each other. A very good solution would be to have three distinctive ship domains, each with its own shape and formula for determination. The three different domains will provide safe distances for three types of obstructions - other vessels, small obstacles, such as buoys, and land and shoal areas. The domain shape is the other parameter that has not experienced a lot of changes recently. In his study of distribution of safe distances for other vessels [4] Lucjan Gucma discovers that in reality ship domains have a varying shape depending on the ship and circumstances. He analyzes the conduct of vessels near Germany and the distances at which they pass each other.

His approach for determining ship domains makes them far more realistic and reliable. His research can be the ground work for the first layer of the formerly proposed 3-layer ship domain. This method or a similar one can also be used when determining the other two types of domains - for single obstructions and for land and shoal areas.

\subsubsection{The issue of communication}

When utilizing EAs for collision avoidance, a vessel is provided with the optimal set of trajectories for all vessels involved. A complication arises when only the own vessel is equipped with EA software. In such a case the navigator will have available the optimum speed and course for all participating vessels, and will know for sure that those trajectories are the most accurate since an algorithm has defined them, but the officers on watch on the rest of the ships will not. They may have their own subjective opinion regarding the developing situation and will never know if their opinion is wrong or not, because they lack information about the future maneuvers of the other ships and they rely solely on their own experience. As the other navigators act on their own accord the evolutionary algorithm on the own ship becomes next to useless and will constantly provide contrasting solutions as the situation develops.

It stands to reason that there must be a way for a vessel to communicate the results of its EA software to the vessels that do not possess any.

One convenient structure for such a communication could be the maritime cloud. If all vessels involved are registered in the maritime cloud then they can easily overlay the optimum trajectories on ECDIS, the same way other vessels' passage plans can be overlaid.

The maritime cloud be used in an alternative way where the EA software can export the results as a user map or an image file, which can be uploaded to the cloud and referenced by the rest of the participants. A user map file can again be loaded 
into ECDIS for easy reference. Nowadays file transfers between ships are even more effective because of the newly introduced systems like Opti BAND by Thru [11] or Binfer [12].

\subsubsection{Issues with delay in response action}

Another minor drawback may be observed in a situation where navigators employ an evolutionary algorithm and are not executing the proposed maneuvers at the initially given times. The proposed solution by the EA may no longer be viable if a vessel delays to alter course or to reduce her speed at the suggested time. As such, the algorithm will automatically begin to calculate a new safe route, which can be entirely different from the previous one, further reducing the odds for a safest maneuver. Even more delay can be obtained if the officer on watch decides to alter course on his own accord. Then the algorithm will continuously restart the calculations based on the changing circumstances and will be unable to provide a plausible solution.

\subsubsection{Complications with different software versions}

One particular disadvantage in using various types of software, which is supposed to interact with vessels other than own ship, presents itself in the face of software version updates. Usually after the initial software is launched, it will feature periodical updates, so it may become better, faster and more reliable. Thus the same software will have different versions on different vessels. The complication with this situation is as follows: Not all vessels will be able to apply or install the latest version of the software. The reasons why a vessel may not have the latest version of the EA software are many, but that is beside the main point. The problem which arises is that different versions of the same software may process data differently and yield various results.

In the following example Vessel A uses Version 2.1 of an EA Algorithm and Vessel B uses the more advanced Version 3.0 of the same algorithm. In addition Vessel B has a slower speed than Vessel A.

Both versions of the algorithm use lane encouragement factors but Version 2.1 operates with more stringent stationary constraints. As the algorithm becomes more advanced, the penalties for stationary constraints have been reduced in the latest version. In the following scenario algorithm Version 2.1 will provide the solution on Fig. 2. Algorithm Version 3.0 will provide the solution on Fig. 3.

As it can be seen, algorithm Version 2.1 will expect Vessel B to start altering course to port to avoid the land mass, while Version 3.0 on vessel B will be displaying various results based on the unexpected course alterations of Vessel A. As a result (Fig. 4) this scenario may lead to dangerous close quarters situation with irreversible consequences.

Other differences between software versions may include a change in the amount of iterations required to reach an acceptable solution. If one algorithm requires more iterations, then the time needed for the calculation will also increase. Computing power on different ships is a factor as well and will differ based on the 
hardware installed. As a result, an EA with fewer iterations or an algorithm, installed on hardware with more computing power will provide a solution to the navigator sooner.

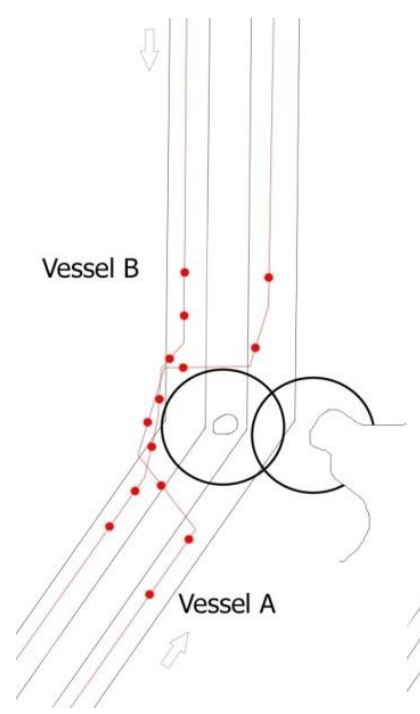

Fig. 2

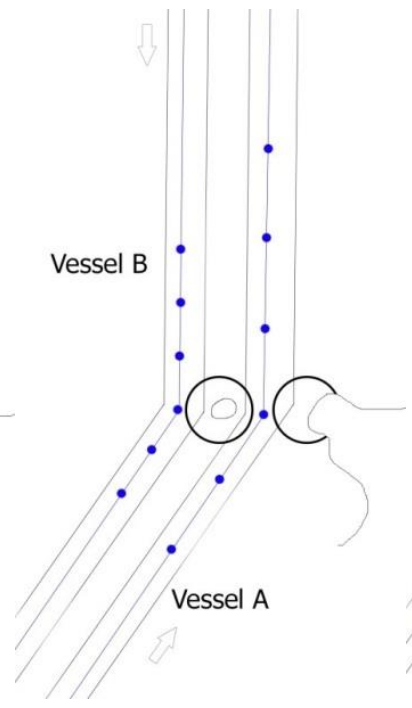

Fig. 3

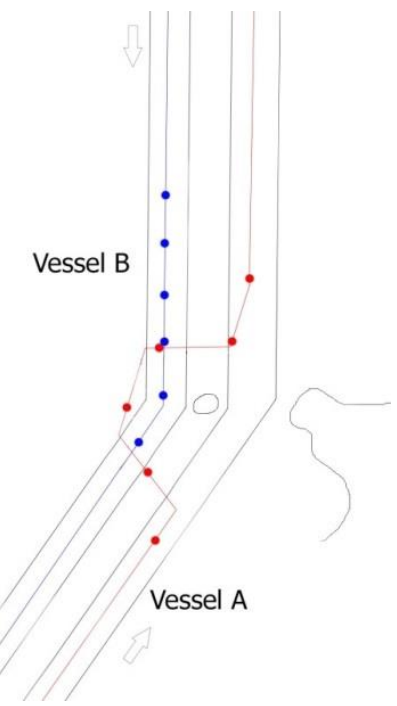

Fig. 4

In a scenario where one algorithm reaches an acceptable solution faster than another and where an officer of the watch promptly alters the course based on the said solution, a slower algorithm on a nearby vessel will not be able to reach an acceptable solution at all, because its software will restart the process every time the base input data shifts with the course alteration of the first vessel.

\subsubsection{Defining the base for ship domains}

Another point to be observed for algorithms which take into account other vessels is the definition of ship's domains and the reliability of the received data.

AIS receives position data from GPS which at times may not be reliable due to solar flares, military practice areas etc. When an AIS unit is receiving wrong position data from GPS then the same wrong position data is transmitted on the VHF frequency to all vessels around. To counter that, a separate module can be included in the algorithm to check AIS position data vs ARPA data. The modern ECDIS has already implemented this under the name of AIS Association. In general, if the target data from the ARPA matches the AIS target data with some margin for error, then both targets are displayed as one. However, if there is a difference between the bearing, range, course or speed of the target above the preset parameter values, the ECDIS displays one target for ARPA and another target for AIS next to it.

The same module can be incorporated in evolutionary algorithms with priority given to ARPA position data, as it does not rely on satellite transmissions. 
However, the solution comes with its own flaws as ARPA data is not $100 \%$ accurate and still allows for errors. A compromise must be established between the two systems to ensure smooth and accurate representation of the targets around the own vessel.

\subsection{Division of the EAs}

A drawback in some EAs $[7,10,13]$ is that they fail to take into account the adverse effects of heavy weather. In this particular case it is safe to divide the evolutionary algorithms into two major categories:

In the first category, there are EAs that take into account heavy weather and are so designed to avoid it to a specified extent. But in their source code there is nothing incorporated regarding avoiding close quarter situations. These solutions direct vessels across oceans, limiting their encounters with foul weather and procure the most optimal route from a safe and cost-effective perspective.

In the second category, there are algorithms that do their best to avoid collisions, land masses and to keep the vessel on the shortest safest route, yet they aren't designed to account for rough seas or gale-force winds. These solutions are designed to navigate vessels in coastal areas and areas with congested traffic and traffic separation schemes, thus finding the shortest path from a time perspective.

\section{Conclusion}

Genetic EAs are perfectly suited for both collision avoidance and passage planning in coastal areas. EAs mitigate the factor of human error in the decision making process and provide reliable results which can be used both by officers and VTS control centers. It is up for debate whether EAs will be implemented on board ships or in the service of VTMIS. Despite some of the drawbacks mentioned in this article, Genetic EAs have a future in the maritime industry. However it is advisable that more time is given for further research, in order to phase out their current deficiencies and perfect their performance.

With regard to passage planning Genetic EAs may not be the best possible solution for a most energy efficient passage plan, as there are other algorithms which take into account a lot more weather factors, but Genetic EAs are the only algorithms that can combine collision avoidance and passage planning in a single real time working module.

\section{References}

1. K u m a r, A., D. Ku m a r, S. J a r i a l. A Review on Artificial Bee Colony Algorithms and Their Applications to Data Clustering. - Cybernetics and Information Technologies, Vol. 17, 2017, No 3, pp. 3-28

2. B a l a b a n o v, T., I. Z a n k i n s k i, M. B a r o v a. Strategy for Individuals Distribution by Incident Nodes Participation in Star Topology of Distributed Evolutionary Algorithms. - Cybernetics and Information Technologies, Vol. 16, 2016, No 1, pp. 80-88. 
3. E i b e n, S. Introduction to Evolutionary Computing. Ch. II. e-Book. 2015, p. 1.

4. G u c m a, L., K. M a r j a n. Examination of Ships Passing Distances Distribution in the Coastal Waters in Order to Build a Ship Probabilistic Domain. Maritime University of Szczecin, Institute of Marine Traffic Engineering, 2012.

5. Guide for Vessel Maneuverability. American Bureau of Shipping. March 2006, pp. 98-99.

6. S z l a p c z y n ski, R. Evolutionary Ship Track Planning within Traffic Separation Schemes Evaluation of Individuals, 2013.

http://www.transnav.eu

7. Szlapczynski, R., J. Szlapczynska. On Evolutionary Computing in Multi-Ship Trajectory Planning. Published Online, 2011. https://www.researchgate.net/publication/257518416

8. G e o r g i e v a, P. Genetic Fuzzy System for Financial Management. - Cybernetics and Information Technologies, Vol. 18, 2018, No 2, pp. 20-35.

9. Y u, X., M. G e n. Introduction to Evolutionary Algorithms. Beijing, 2010, p. 7.

10. Zha o, Y.-x., W. Li. Improved Differential Evolution Algorithm for Maritime Collision Avoidance Route Planning. - Abstract and Applied Analysis, Vol. 2014.

11. With OptiBAND You Can. 12.11.2017. http://www.thruinc.com/optiband/

12. Binfer Moves Your Data without Ever Storing It on Any Third Party Systems. 12.11.2017. https://www.binfer.com/

13. Theja, P., S. K. B abu. Evolutionary Computing Based on QoS Oriented Energy Efficient VM Consolidation Scheme for Large Scale Cloud Data Centres. - Cybernetics and Information Technologies, Vol. 16, 2016, No 2, pp. 97-112.

Received: 24.04.2018; Second Version: 13.11.2018; Accepted: 10.12.2018 